\title{
Boundary Value Problems and Approximate Solutions
}

\section{Gebreslassie Tesfy, Venketeswara Rao J*, Ataklti Araya and Daniel Tesfay}

Department of Mathematics, College of Natural and Computational Scineces, Mekelle University, Mekelle, Tigrai, Ethiopia (*drvekateswararao@gmail.com)

\begin{abstract}
In this paper, we discuss about some basic things of boundary value problems. Secondly, we study boundary conditions involving derivatives and obtain finite difference approximations of partial derivatives of boundary value problems. The last section is devoted to determine an approximate solution for boundary value problems using Variational Iteration Method (VIM) and discuss the basic idea of He's Variational Iteration Method and its applications.
\end{abstract}

Key Words: Boundary value problem, Boundary conditions, Variational Iteration Method, He's Variational Iteration Method, Finite difference method, Standard 5-point formula, Iteration method, Relaxation method and standard analytic method.

\section{Introduction}

Solutions of Boundary Value Problems can sufficiently closely be approximated by simple and efficient numerical methods. Among these numerical methods are finite difference method, standard 5-point formula, iteration method, relaxation method and standard analytic method. But here the finite-difference method and Variational Iteration Method will be considered.

Boundary value problems arise in several branches of physics as any physical differential equation will have them. Problems involving the wave equation, such as the determination of normal modes, are often stated as boundary value problems. A large class of important boundary value problems are; the Sturm-Liouville problems. The analysis of these problems involves the Eigen functions of a differential operator. Consider the second linear boundary problem: $\frac{d^{x} y}{d x^{2}}+f(x) \frac{d y}{d x}+g(x) y(x)=r(x)$

with the boundary conditions: $y\left(\kappa_{Q}\right)=$ a and $y\left(\kappa_{n}\right)=b$

There exist several methods to solve second order boundary value problem. One of these is the finite difference method, which is most popular. Amann (1986) contributed to Quasilinear Parabolic systems under nonlinear boundary conditions. Deng and Levine (2000) studied about the role of critical exponents in blow-up theorems. Friedman (1967) made an introduction to partial differential equations of parabolic type. Friedman and McLeod (1985) developed the blow-up of positive solutions of semi-linear heat equations. Keller (1969) studied elliptic 
boundary value problems suggested by nonlinear diffusion process. Lady'zenskaja et al. (1988) developed the concept of linear and quasilinear equations of parabolic type. Le Roux (1994) made a semi-discretization in time of nonlinear parabolic equations with blowup of the solutions. Le Roux (2000) derived a numerical solution of nonlinear reaction diffusion processes. Levine (1990) identified the role of critical exponents in blow-up theorems. Mochizuki and Suzuki (1997) find a critical exponents and critical blow up for quasi-linear parabolic equations. Qi (1991) studied the asymptotics of blow-up solutions of a degenerate parabolic equations. Samarskii et al. (1995) observed a blow-up in quasilinear parabolic equations. Sperp (1980) studied the maximum principles and their applications. Zhang (1997) achieved on blow-up of solutions for a class of nonlinear reaction diffusion equations.

\section{METHODOLOGY}

The finite difference method for the solution of a two point boundary value problem consists in replacing the derivatives present in the differential equation and the boundary conditions with the help of finite difference approximations and then solving the resulting linear system of equations by a standard method.

It is assumed that $y$ is sufficiently differentiable and that a unique solution of (1.1) exists. Problems of this kind are commonly encountered in plate-deflection theory and in fluid mechanics for modeling viscoelastic and inelastic flows (Usmani, 1977a; Usmani, 1977b; Momani, 1991). Usmani (1977a, 1977b) discussed sixth order methods for the linear differential equation $y^{4}+P(x) y=q(x)$ subject to the boundary conditions $y(a)=A_{y} y^{\prime \prime}(a)-A_{y} y(b)=E_{2 y} y^{\prime \prime}(b)=E_{2}$. The method described by Usmani (1977a) leads to five diagonal linear systems and involves $p^{\prime}, p^{\prime \prime}, q^{\prime}, q^{\prime \prime}$ at $a$ and $b$, while the method described in Usmani (1977b) leads to nine diagonal linear systems.

Ma and Silva (2004) adopted iterative solutions for (1.1) representing beams on elastic foundations. Referring to the classical beam theory, they stated that if $u=u(x)$ denotes the configuration of the deformed beam, then the bending moment satisfies the relation $M=-E I u^{\prime \prime}$, where $E$ is the Young modulus of elasticity and $I$ is the inertial moment. Considering the deformation caused by a load $f=f(x)$, they deduced, from a free-body diagram, that $f=-v^{t}$ and $v=M^{i}=-E I u^{m I}$, where $v$ denotes the shear force. For $u$ representing an elastic beam of 
length $L=1$, which is clamped at its left side $x=0$, and resting on an elastic bearing at its right side $x=1$, and adding a load $f$ along its length to cause deformations, Ma and Silva [2004] arrived at the following boundary value problem assuming an $E I=1$ :

$u^{(t w)}(x)=f(x, u(x)), 0<x<1$,

the boundary conditions were taken as

$u(0)=u^{t}(0)=0_{r}$

$u^{t t}(1)=0, u^{t t}(1)=g(u(1))$

where $f \in \mathscr{E}([, 1] \times E)$ and $g \in C(\mathrm{R})$ are real functions. The physical interpretation of the boundary conditions is that $u^{t u}(1)$ is the shear force at $x=1$, and the second condition in (1.5)

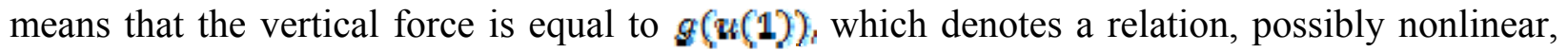
between the vertical force and the displacementu(1). Furthermore, since $u^{\prime \prime}(1)-0$ indicates that there is no bending moment at $x=1$, the beam is resting on the bearing $g$.

Solving (1.3) by means of iterative procedures, Ma and Silva (2004) obtained solutions and argued that the accuracy of results depends highly upon the integration method used in the iterative process. With the rapid development of nonlinear science, many different methods were proposed to solve differential equations, including boundary value problems (BVPS). In this paper, it is aimed to apply the variational iteration method proposed by He (1999) to different forms of (1.1) subject to boundary conditions of physical significance.

\section{BOUNDARY VALUE PROBLEMS}

3.1 Definition: A boundary value problem is a differential equation together with a set of additional restraints, called the boundary conditions.

3.2 Definition: A solution to a boundary value problem is a solution to the differential equation which also satisfies the boundary conditions.

3.3 Note: Using the Taylor's series, we have

$y(x+h)=y(x)+h y^{\prime}(x)+\frac{h^{n}}{2 i} y^{\prime \prime}(x)+\frac{h^{2}}{g !} y^{\prime \prime \prime}(x)+\cdots$

$y(x-h)=y(x)-h y^{t}(x)+\frac{h^{n}}{2 i} y^{\prime \prime}(x)-\frac{h^{2}}{a t} y^{\prime \prime \prime}(x)+\cdots$

$n y^{k}(x)=\frac{y(x+h)-y(x)}{h}-\frac{k}{2} y^{n t}(x) \ldots$ 


$$
=\frac{x(x+h)-x(\alpha)}{\hbar}+O(h)
$$

This is the forward difference approximation for $y^{p}(x)$. Also we have

$y^{t}(x)=\frac{y(x)-y(x-h)}{h}+O(h)$

This gives the back-ward difference approximation for $y^{\prime}(z)$. Then the central difference approximation for $y^{\prime}(x)$ is obtained by subtracting (2.2) from (2.1);

$$
\begin{aligned}
& y(x+h)-y(x-h)-2 h y^{\prime}(x)+\frac{2 h^{2}}{3 !} y^{\prime \prime \prime}(x)+\cdots \\
& \Rightarrow y^{t}(x)=\frac{y(x+h)-y(x-h)}{2 h}-\frac{h}{3 !} y^{\prime \prime \prime}(x) \ldots \\
& =\frac{y(x+h)-y(x-h)}{2 h}+o\left(h^{2}\right)
\end{aligned}
$$

This gives us a better approximation to $y^{\prime}(x)$ as compared to (2.3) or (2.4). Further adding (2.1) and (2.2), we get;

$$
\begin{aligned}
& y(x+h)+y(x-h)=2 y(x)+h^{2} y^{\prime \prime}(x)+\frac{2 h^{4}}{4 !} y^{w}(x)+\cdots \\
& \Rightarrow \frac{y(x+h)-2 y(x)+y(x-h)}{h^{2}}=y^{\prime}(x)+\frac{2 h^{2}}{4 !} y^{w}(x)+\cdots \\
& \Rightarrow y^{\prime \prime}(x)=\frac{y(x-h)-2 y(x)+y(x+h)}{\hbar^{2}}+e\left(h^{2}\right)
\end{aligned}
$$

Similarly we can obtain finite-difference approximations of higher derivatives. To solve the boundary-value problem given by $\frac{a^{2} y}{d x^{2}}+f(x) \frac{d y}{d x}+g(x) y(x)=r(x)$ with the boundary conditions: $y\left(x_{0}\right)=a$ and $y\left(x_{n}\right)=b$, we divide the range $\left[x_{0}, x_{n}\right]$ in to n-equal sub intervals of width $h$ so that $x_{f}-x_{0}+t k_{k} \quad l=1,2,3, \ldots \ldots z$. The corresponding value of $y$ are then given by $y\left(x_{t}\right)=y_{t}=y\left(x_{0}+t h\right)_{1} t=1,2,3_{t} \ldots \ldots n$ n.

Now using equations (2.5) and (2.6); values of $y^{\prime}(x)$ and $y^{t \prime}(x)$ at the points $x=x_{i}$ can be written as:

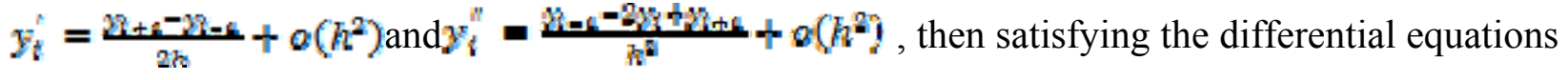
at the point $x=x_{i}, y=y_{t}$ we get, $y_{i}^{\prime \prime}+f_{i} y_{t}^{\prime}+g_{i} y_{t}=r_{t}$

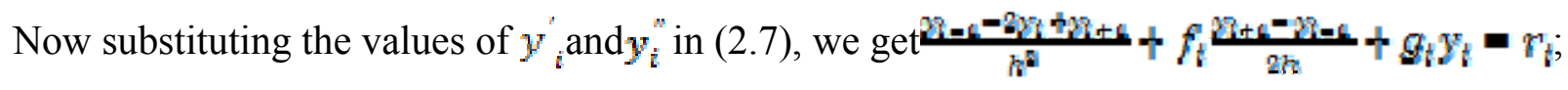


$t=1,2,3_{n} \ldots \ldots \ldots(n-1)$ where $y_{t}=y\left(x_{t}\right) g_{t}=g\left(x_{t}\right)$ etc. multiplying throughout by $h^{2}$ and then simplifying, we get

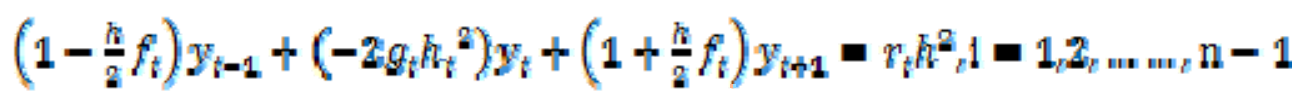

Where $y_{0}=a$ and $y_{n}=b$.

Now equation (2.8) under the conditions (2.9) gives rise to a tridiagonal system which can be easily solved by the model method.

Example 1: Solve numerically the equation $y^{\prime \prime}+y+1=0$, with boundary conditions $y=0$ when $x=0$, and $y=0$ when $x=1$.

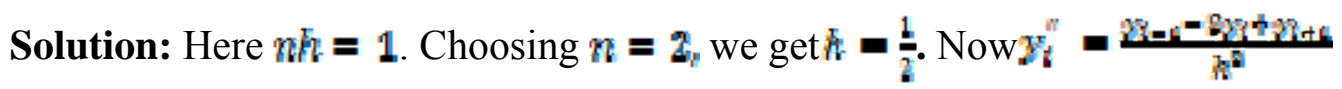
Putting this value in the given equation, we get $\frac{25-2 x^{2}+3 x+4}{h^{2}}+y_{i}+1=0$

$$
\begin{aligned}
& \text { - } \\
& \left(x_{0}, y_{0}\right) \quad\left(x_{1}, y_{1}\right) \quad\left(x_{2}, y_{2}\right)
\end{aligned}
$$

Figure 1. Mesh points of $y^{\prime \prime}+y+1=\emptyset$ when $n=\mathbf{2}$

$$
\Rightarrow y_{i-1}=\left(2-h^{2}\right) y_{t}+y_{i+1}=-h_{i}^{2} t=1,2_{i} \ldots,(n-1)
$$

Putting $n=2$, we get $i=1 \Rightarrow y_{0}-\left(2-\frac{1}{4}\right) y_{1}+y_{2}=-\frac{1}{4}$ but $y_{6}=0$ and $y_{2}=0$

$$
\left.n-\left(\frac{7}{4}\right) y_{1}=-\frac{1}{4} \quad \Rightarrow y_{1}=\frac{1}{\gamma}=0.14285 \ldots \text { But } y_{1}=y(0.5)=0.14285 \ldots{ }^{*}\right)
$$

Again solving the given equation analytically by standard method, we get

$$
y\left(\frac{1}{2}\right)=0.13949 \ldots \ldots(* *) \text {. }
$$

Inspections $(*)$ and $(* *)$ shows that the finite-difference solution for $y\left(\frac{1}{2}\right)$ is about $2.4 \%$ too large.

\section{BOUNDARY CONDITIONS INVOLVING A DERIVATIVE}

4.1 Definition: The central difference $\$ y$ of $y$ is defined by

$$
\delta y\left(x_{f}\right)=y\left(x_{f}+\frac{1}{2} h\right)-y\left(x_{f}-\frac{1}{2} h\right) .
$$

Higher-order differences are defined recursively by:

$$
\delta^{m+1} y\left(x_{f}\right)=\delta\left[\delta^{m} y\left(x_{f}\right)\right]=\delta^{m} y\left(x_{l}+\frac{1}{2} h\right)-\delta^{m} y\left(x_{l}-\frac{1}{2} h\right) .
$$


In particular, the second central difference may be written

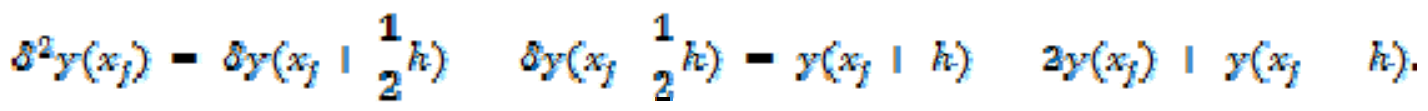

The differential equation $y^{n}+r(x) y=f(x)_{t} a \alpha_{n} x \alpha_{n} b_{v}$

may be associated with boundary conditions involving the first derivative of the solution.

Suppose, for example, that we are given real numbers $0, A$ and B. Consider the differential equation (3.1) together with the boundary conditions $y^{b}(a)-x y(\alpha)-A_{\ell} y(b)=E$

The condition at $x=a$ may be approximated in various ways; we shall introduce an extra mesh point $x_{-1}$ outside the interval and use the approximate version $\frac{z_{-1}-y_{-4}}{2 n}-\propto Y_{\alpha}$.

This gives $y_{-1}=y_{1}-2 h-\propto Y_{0}^{N}-2 h A$. Writing the same central difference approximation

$$
\frac{\delta^{2} Y_{t} h^{n}}{n^{2}}+r_{l} Y_{l}=f_{j} t=1_{l}, 3_{n+1} n-1 \text {, }
$$

But now for $f=0,1, z_{n}, n-1$ we can eliminate the extra unknown $y_{-1}$ from the equation at $j=0$ to give $\left[\frac{2\left(1+x_{h}^{2}\right.}{h^{2}}+s_{0}\right] Y_{0}-\frac{2}{h^{2}} x_{1}-f_{0}-\frac{2}{h} A$ together with (3.1), for $f=1,2, \ldots, n-1$ we now have a system of $n$ equations for the unknowns $Y_{1}, t=0,1_{1}, \mathbf{z}_{l} \ldots-1$ there are one more equation and one more unknown.

4.2 Theorem: Suppose that $y^{2} c^{a}\left(x-h_{r} x+h\right)$ then, there exists a real number $x$ in $\left(x-h_{i} x+h\right)$ such that $\frac{2(x+h)-x\left(x-h_{i}\right)}{2 \hbar}=y^{t}(x)+\frac{1}{6} h^{2} y^{t u}(x)$

Proof: Taylor's Theorem shows that there exist $x_{1} \in\left(x-k_{r} x\right)$ and $x_{2} \in\left(x_{r} x+k\right)$ such that $y(x-h)=y(x)-h y^{t}(x)+\frac{1}{2} h^{2} y^{n}(x)-\frac{1}{6} h^{2} y^{n \prime}\left(x_{1}\right)$ $y(x+h)=y(x)+h y^{6}(x)+\frac{1}{2} h^{2} y^{n}(x)+\frac{1}{6} h^{8} y^{n n}\left(x_{1}\right)$

We subtract the first equality from the second, and note that the approximation to $y^{t}(x)$ at

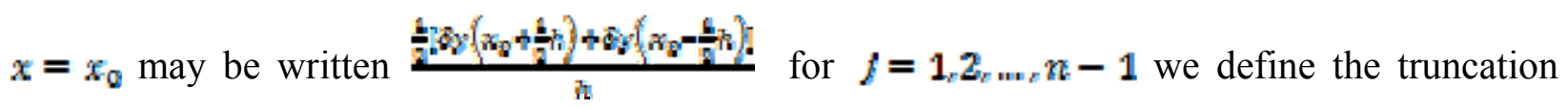
error $T_{j}$. In addition, since we shall now also incur an error in the approximation of the boundary condition at $x=a$, we define

$T_{0}=\left[\frac{2\left(1+\omega_{h}^{2}\right.}{h^{2}}+r_{0}\right] y(0)-\frac{2}{h^{2}} y(h)-f_{0}+\frac{2}{h} A$. 


\section{OBTAINING FINITE DIFFERENCE APPROXIMATIONS OF PARTIAL DERIVATIVES}

Let the x-y plane be divided into a network of rectangles of side $\Delta_{w}=h$ and $\lambda_{y}=h$ by drawing the set of lines $x=$ th $(t-1,2, \ldots)$ and $y=t k\left(f=1_{l} \mathbf{2}_{p} \ldots\right)$, The points of intersection of these lines are called mesh points (lattice points or grid points).

\begin{tabular}{|c|c|c|c|}
\hline & & $(x, y+k)$ & \\
\hline & $(x-h, y)$ & $\begin{array}{l}(t, f+1) \\
(x, y)\end{array}$ & $(x+h, y)$ \\
\hline$=k$ & & & \\
\hline & $(t-1, t)$ & $\begin{array}{l}(t, f) \\
(x, y=k)\end{array}$ & $(t+1, f)$ \\
\hline & & $(t, j-1)$ & \\
\hline 0 & & & \\
\hline
\end{tabular}

Figure 2. Mesh points (lattice points or grid points).

Then we have the finite difference approximations for the partial derivatives in $\mathrm{x}$-direction, as

$\frac{\partial u}{\partial x}=\frac{w(x+h, y)-w(x, y)}{h}+\sigma(h)=\frac{w(x, y)-w(x-h y)}{\hbar}+\sigma(h)=\frac{w(x+h y)-w(x-h, y)}{2 h}+O\left(h^{2}\right)$

$\frac{g^{2} u}{\partial x^{2}}=\frac{u(x-s h, y)-2 u(x, y)+u\left(x+h_{r} y\right)}{h^{2}}+\theta\left(h^{2}\right)$

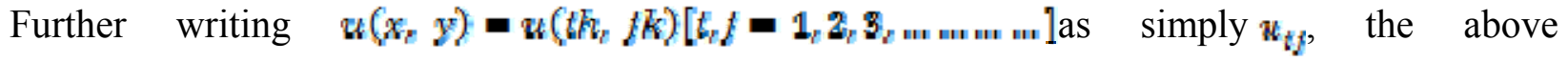
approximations are reduced to:

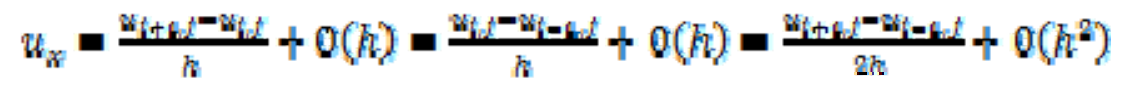

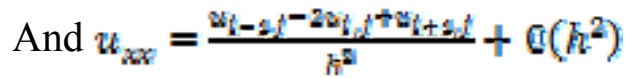

And similarly we have the approximations for the derivatives w.r.to y:

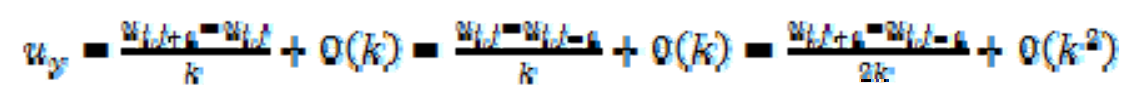

And $u_{y y}=\frac{w_{b, t-1}-2 u_{i, t}+w_{t, j+2}}{k^{2}}+0\left(k^{2}\right)$ 


\section{AN APPROXIMATE SOLUTION FOR BOUNDARY VALUE PROBLEMS}

6.1 Definition: The general form of the equation for a fixed positive integer $n, n \geq 2$ is a

differential equation of order $2 n: y^{2 n}+f(x, y)=0$

Subject to the boundary conditions $y^{2}(a)=A_{2 y n} y^{(2 n}(b)=E_{2 p} l=0(1) n-1$,

where $-\infty<a \leq x \leq b<\infty, A_{2 \gamma}, B_{2 p} f=0,1,2, \ldots, n-1$ are finite constants.

\subsection{Basic idea of He's variational iteration method}

To clarify the basic ideas of He's VIM, the following differential equation is considered:

$E[u(t)]+N[u(t)]=g(t)$,

where $L$ is a linear operator, $N$ is a nonlinear operator, and $g(t)$ is an inhomogeneous term.

According to VIM, a correction functional could be written as follows:

$u_{n+1}(t)=u_{n}(t)+\int_{a}^{2} \lambda(\tau)\left(L\left(u_{n}(\tau)+N u_{n}(\tau)-g(\tau)\right) d r\right.$

where $\lambda$ is a general Lagrange multiplier which can be identified optimally via the variational theory and the subscript $n$ indicates the $n$th approximation and $\boldsymbol{V}_{\mathrm{m}}^{\prime}$ is considered as a restricted variation, that is, $8 \pi_{\mathrm{n}}^{\infty}=0$.

For fourth-order boundary value problem with suitable boundary conditions, Lagrangian multiplier can be identified by substituting the problem into (5.4), upon making it stationary leads to the following:

$$
\begin{array}{r}
\frac{d^{4}}{d x^{4}} \lambda=0 \\
-\lambda^{m+1}+1 / \tau-x=0 \\
\lambda^{m} / \tau \omega_{x}=0 .
\end{array}
$$

Solving the system of (5.5) yields

$$
\lambda=\frac{1}{6}(x-x)^{3}
$$

and the variational iteration formula is obtained in the form

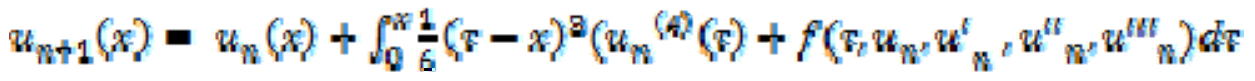

\subsection{The Applications of Variational Iteration Method}

In this section, the Variational Iteration Method is applied to different forms of the fourth-order boundary value problem introduced in through (5.1).

Example 1: Consider the following linear boundary value problem: 
$u^{(4)}(x)=4 e^{x}+u(x), 0<x<1$

subject to the boundary conditions

$w(0)=1, w^{t}(0)=\mathbf{2}, w(1)=\mathbf{2}, \quad u^{t}(0)=3 \theta$

The exact solution for this problem is

$u(x)=(1+x) e^{x}$

According to (5.7), the following iteration formulation is achieved:

$u_{n+1}(x)=u_{n}(x)+\int_{0}^{x} \frac{1}{6}(\tau-x)^{a}\left(u_{n}(4)-u_{n}(\tau)-4 e^{T}\right) d r$.

Now it is assumed that an initial approximation has the form

$w_{0}(x)=a x^{2}+b x^{2}+c x+d_{v}$

where $a, b, c$, and $d$ are unknown constants to be further determined.

By the iteration formula (5.11), the following first-order approximation may be written:

$$
\begin{aligned}
& u_{1}(x)=u_{0}(x)+\int_{0}^{x} \frac{1}{6}(\tau-x)^{2}\left(u_{0}{ }^{4}(\tau)-u_{0}(\tau)-4 e^{v}\right) d \tau \\
& =a x^{2}+b x^{2}+c x+d+\int_{0}^{x} \frac{1}{6}(r-x)^{2}\left(-a r^{2}-b r^{2}-c r-d^{2}-4 e^{2}\right) d r \\
& =\frac{1}{840} a x^{5}+\frac{1}{260} b x^{6}+\frac{1}{120} c x^{8}+\frac{1}{24} d x^{4}+\left(-\frac{2}{2}+a\right) x^{2}+(b-2) x^{2}+(c-4) x+4 a^{x}+d^{2}-4
\end{aligned}
$$

Incorporating the boundary conditions (5.9), into $k_{1}(x)$, the following coefficients can be obtained:

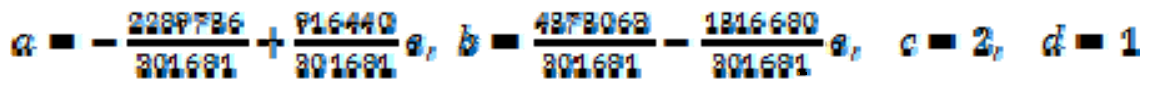

Therefore, the following first-order approximate solution is derived:

$$
\begin{aligned}
& u_{1}(x)=\left(-\frac{27259}{301681}+\frac{1091}{301681} \varepsilon\right) x^{7}+\left(\frac{1525021}{36201720}-\frac{4713}{3016810} \varepsilon\right) x^{6}+\frac{1}{60} x^{6}+\frac{1}{24} x^{4} \\
& +\left(-\frac{7472630}{905043}+\frac{916440}{301681} \mathrm{e}\right) x^{2}+\left(\frac{3971701}{301681}-\frac{1516680}{301681} \mathrm{e}\right) x^{2}+2 x-3 \\
& +4 e^{x} \text {. }
\end{aligned}
$$

Comparison of the first-order approximate solution with exact solution is tabulated in table 1 , showing a remarkable agreement. 
Table 1. Comparison of the first-order approximate solution with exact solution.

\begin{tabular}{|l|l|l|l|}
\hline$x$ & $U_{E}$ & $U_{1}$ & Error \\
\hline 0 & 1.000000000 & 1.000000000 & $0.0000 E+000$ \\
\hline 0.1 & 1.215688010 & 1.215681524 & $6.4860 E-006$ \\
\hline 0.2 & 1.465683310 & 1.465660890 & $2.2420 E-005$ \\
\hline 0.3 & 1.754816450 & 1.754773923 & $4.2527 E-005$ \\
\hline 0.4 & 2.088554577 & 2.088492979 & $6.1598 E-005$ \\
\hline 0.5 & 2.473081906 & 2.473007265 & $7.4641 E-005$ \\
\hline 0.6 & 2.915390080 & 2.915312734 & $7.7346 E-005$ \\
\hline 0.7 & 3.423379602 & 3.423312592 & $6.7010 E-005$ \\
\hline 0.8 & 4.005973670 & 4.005929404 & $4.4266 E-005$ \\
\hline 0.9 & 4.673245911 & 4.67322989 & $1.6020 E-005$ \\
\hline 1.0 & $2 \mathrm{e}$ & $2 e$ & $0.0000 E+000$ \\
\hline
\end{tabular}

Similarly, the following second-order approximation is obtained:

$$
\begin{aligned}
& u_{2}(x)=u_{1}(x)+\int_{0}^{x} \frac{1}{6}(\tau-x)^{2}\left(u_{1}(\tau)-u_{1}(\tau)-4 \theta^{\tau}\right) d r \\
& =\frac{1}{6682900} a x^{11}+\frac{1}{1244400} b x^{10}+\frac{1}{262980} \operatorname{cs}^{8}+\frac{1}{40220} d x^{9}+\left(\frac{1}{840} a-\frac{1}{1260}\right) x^{7}+ \\
& \left(\frac{1}{260} b-\frac{1}{190}\right) x^{6}+\left(-\frac{1}{20}+\frac{1}{120} c\right) x^{8}+\left(-\frac{1}{6}+\frac{1}{24} \alpha^{2}\right) x^{4}+\left(-\frac{4}{2}+a\right) x^{2}+ \\
& (b-4) x^{2}+(c-8) x-8+8 e^{x}+\alpha_{l}
\end{aligned}
$$

$a=-\frac{12706529114180}{681628862391}+\frac{85535681616000}{12042109902241} \epsilon, \quad \varepsilon=2$

$b=\frac{8416302814865}{227209620797}-\frac{157452726614400}{12042109902241} \theta_{0} \quad d=1$

Therefore, the second-order approximate solution may be written as 


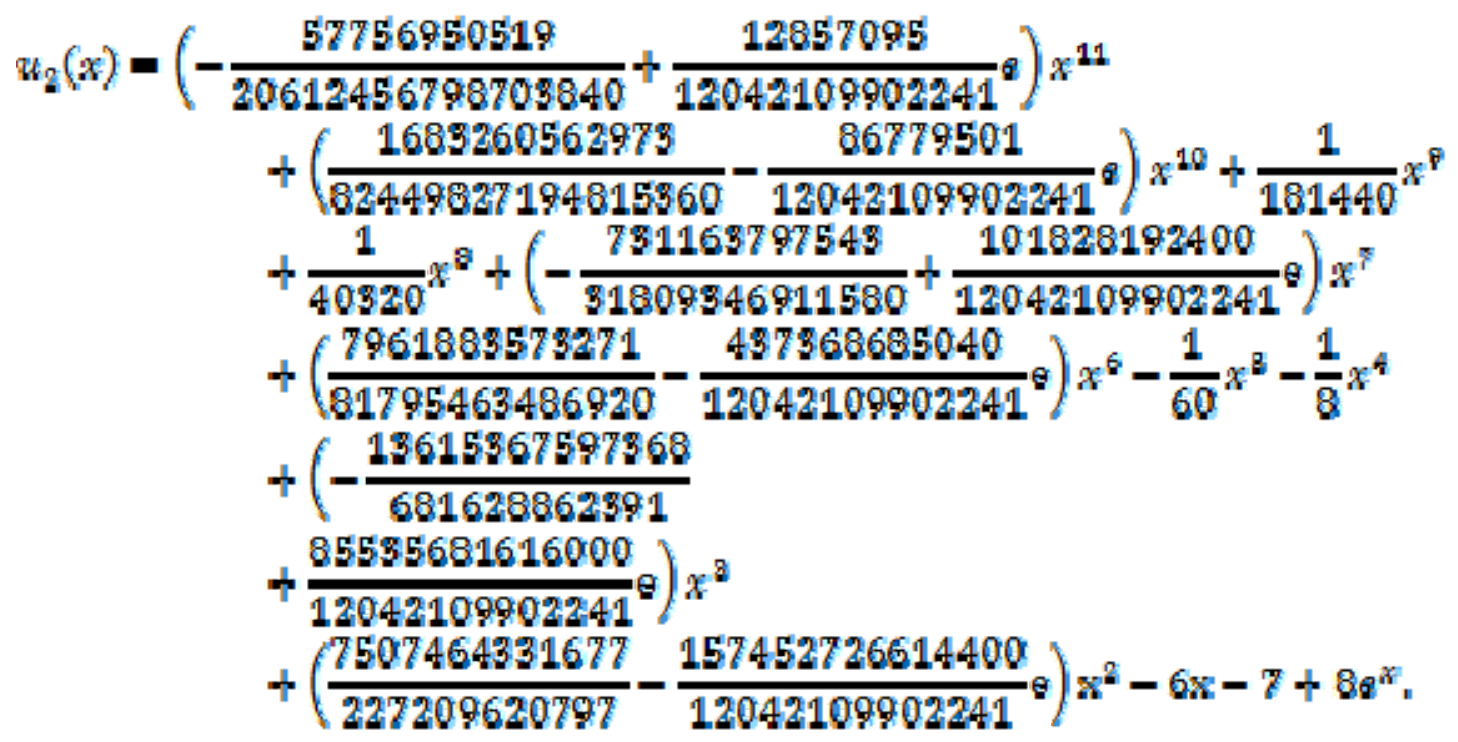

Again, the obtained solution is of distinguishing accuracy, as indicated in table 2 below.

Table 2. Comparison of the second-order approximate solution with exact solution.

\begin{tabular}{|l|l|l|l|}
\hline$x$ & $U_{E}$ & $U_{2}$ & Error \\
\hline 0 & 1.000000000 & 1.000000000 & $0.0 E+000$ \\
\hline 0.1 & 1.215688010 & 1.215688008 & $2.0 E-009$ \\
\hline 0.2 & 1.465683310 & 1.465683305 & $5.0 E-009$ \\
\hline 0.3 & 1.754816450 & 1.754816444 & $6.0 E-009$ \\
\hline 0.4 & 2.088554577 & 2.088554566 & $1.1 E-008$ \\
\hline 0.5 & 2.473081906 & 2.473081902 & $4.0 E-009$ \\
\hline 0.6 & 2.915390080 & 2.915390064 & $1.6 E-008$ \\
\hline 0.7 & 3.423379602 & 3.423379600 & $2.0 E-009$ \\
\hline 0.8 & 4.005973670 & 4.005973650 & $2.0 E-008$ \\
\hline 0.9 & 4.673245911 & 4.673245930 & $1.9 E-008$ \\
\hline 1.0 & $2 \mathrm{e}$ & $2 e$ & $0.0 E+000$ \\
\hline
\end{tabular}

\section{CONCLUSION}

This study showed that the finite difference method for the solution of a two point boundary value problem consists in replacing the derivatives present in the differential equation and the boundary conditions with the help of finite difference approximations and then solving the resulting linear system of equations by a standard method. 
The Variational Iteration Method is remarkably effective for solving boundary value problems. A fourth-order differential equation with particular engineering applications was solved using the VIM in order to prove its effectiveness. Different forms of the equation having boundary conditions of physical significance were considered.

Comparison between the approximate and exact solutions showed that one-iteration is enough to reach the exact solution. Therefore the Variational Iteration Method is able to solve partial differential equations using a minimum calculation process. This method is a very promoting method, which promises to find wide applications in engineering problems.

\section{REFERENCES}

Amann, H. 1986. Quasilinear Parabolic systems under nonlinear boundary conditions, Arch. Rational Mech. Anal., 92:153-192.

Deng, K \& Levine, H. A. 2000. The role of critical exponents in blow-up theorems: The Sequel, J. Math. Anal. Appl., 243:85-126.

Friedman, A. 1967. Partial differential equations of parabolic type. Prentice-Hall.

Friedman, A \& McLeod, J.B. 1985. Blow-up of positive solutions of semi-linear heat equations, Indiana Math. J., 34:425-447.

He, J.H. 1999. Variational iteration method a kind of nonlinear analytical technique: some examples. International Journal of Non-Linear Mechanics, 34(4): 699-708.

Keller, H.B. 1969. Elliptic boundary value problems suggested by nonlinear diffusion process. Arch. Rational. Mech. Anal., 35:363-381.

Lady`zenskaja, O. A., Ural'ceva, N. N \& Solonnikov, V. A. 1988. Linear and quasilinear equations of parabolic type, AMS Translations of Math. Monographs, 23.

Le Roux, M.N. 1994. Semidiscretization in time of nonlinear parabolic equations with blowup of the solutions. SIAM J. Numer. Anal., 31:170-195.

Le Roux, M.N. 2000. Numerical solution of nonlinear reaction diffusion processes. SIAM J. Numer. Anal., 37(5):1644-1656.

Levine, H. A. 1990. The role of critical exponents in blow-up theorems. SIAM Reviews, 32: 262288.

Ma, T. F \& Da Silva, J. 2004. Iterative solutions for a beam equation with nonlinear boundary conditions of third order. Applied Mathematics and Computation, 159(1):11-18. 
Mochizuki, K \& Suzuki, R. 1997. Critical exponents and critical blow up for quasi-linear parabolic equations. Isreal J. Math., 98:141-156.

Momani, S. M. 1991. Some problems in non-Newtonian fluid mechanics, Ph.D. thesis, Wales University, Wales, UK.

Qi, Y.W. 1991. The asymptotics of blow-up solutions of a degenerate parabolic equations. $J$. Appl. Math. Phys.(ZAMP), 42:488-496.

Samarskii, A.A., Galaktionov, V.A., Kurdyumov, S.P \& Mikhailov, A.P. 1995. Blow-up in Quasilinear Parabolic Equations. Walter de Gruyter, Berlin (English translation), Nauka, Moscow.

Sperp, R.P. 1980. Maximum Principles and their Applications. Academic Press, NewYork.

Usmani, R. A. 1977a. On the numerical integration of a boundary value problem involving a fourth order linear differential equation. BIT, 17(2):227-234.

Usmani, R. A. 1977b. An $\mathrm{O}\left(\mathrm{h}^{6}\right)$ finite difference analogue for the solution of some differential equations occurring in plate deflection theory. Journal of the Institute of Mathematics and Its Applications, 20(3):331-333.

Zhang, H. 1997. On blow-up of solutions for a class of nonlinear reaction diffusion equations. Chinese J. Math., 17:482-486. 